\title{
Role of early surgical intervention in outcome and prognosis of spontaneous cerebellar hemorrhage with intraventricular extension
}

\author{
Jemesh Singh Maharjan' \\ ${ }^{1}$ Department of Neurosurgery, Norvic International Hospital, Kathmandu, Nepal
}

\author{
Correspondence: \\ Dr. Jemesh Singh Maharjan \\ Department of Neurosurgery \\ Norvic International Hospital \\ Phone - 9849156669 \\ Email - jemesh.s.maharjan@gmail.com
}

\begin{abstract}
Spontaneous cerebellar hematomas represent 5 to $13 \%$ of all cases of spontaneous intracranial hemorrhage. The main controversy involves deciding which cases require surgical evacuation of the hematoma versus other options, such as ventricular drainage only or conservative treatment. Furthermore, because the clinical course is variable in some cases, timing of such treatment should be carefully considered. The duration from the onset of hemorrhage also plays an important role in prognosis and recovery of the patient. Both the clinical presentation and subsequent course vary among cases. Unpredictable rapid deterioration in consciousness levels has been recognized. The majority of patients with such decline in consciousness experience the deterioration primarily within $72 \mathrm{hrs}$ after onset4. Acute presentation was observed to be correlated with poor outcomes. ${ }^{2}$ In our report, the first case presented with sudden onset of headache in the right frontal region of head with vertigo. He came to hospital within 6 hours of onset. However, the second case had an onset of symptoms around 72 hours before the presentation.
\end{abstract}

Key Words: cerebellar hemorrhage, intraventricular extension, spontaneous, surgery

$\mathrm{T}$ The posterior cranial fossa $(\mathrm{PCF})$ is the deepest and most confined space in the skull. It has a limited ability to accommodate an expansion of its contents. ${ }^{1}$ PCF hemorrhage (PCFH) accounts for between 9 and 15 $\%$ of all intracranial hemorrhage in Europe and North America. When left to its natural course, it can result in life-threatening complications such as compression of the pons and medulla, resulting in impaired consciousness, respiratory failure, and lower cranial nerve dysfunction. Acute hydrocephalus may also result due to the obstruction of the fourth ventricle leading to herniation of the posterior cranial fossa contents1. Spontaneous cerebellar hematomas represent 5 to $13 \%$ of all cases of spontaneous intracranial hemorrhage. These hematomas are associated with high mortality rates of 20 to $75 \%$, irrespective of the mode of treatment, irrespective of the mode of treatment, with higher values being reported for the pre-computed tomography era. Current overall surgical mortality rates remain up to 20 to $50 \%$. The depressed level of consciousness in cases of cerebellar hematomas could be attributable to hydrocephalus, direct brain- stem compression by the hematoma and surrounding swelling, or both. The decisions regarding surgical treatment are determined by the size of the hematoma and other factors, such as the presence of hydrocephalus, the degree of basal cisternal compression, and the location of the hematoma. ${ }^{2}$ The main controversy involves deciding which cases require surgical evacuation of the hematoma versus other options, such as ventricular drainage only or conservative treatment. Furthermore, because the clinical course is variable in some cases, the timing of such 
treatment should be carefully considered. Surgical evacuation of posterior fossa hematomas is associated with morbidity and mortality risks. Simple drainage of hydrocephalus may be ineffective. There is a group of patients for whom conservative management is appropriate. ${ }^{2}$ The duration from the onset of hemorrhage also plays an important role in prognosis and recovery of the patient. We're assessing the role of early management, surgical or conservative in the outcome of patients with spontaneous cerebellar hemorrhage.

\section{Case Presentation}

\section{Case 1:}

A 64-year Male presented with sudden onset headache over right frontal region and dizziness room spinning type for duration of an hour. There was no history of loss of consciousness, vomiting, paresis or paralysis of any body parts, abnormal body movements, tinnitus, aural fullness, sweating. He doesn't give a history of recent trauma to head. The patient is non-hypertensive, non-diabetic and has no other chronic illnesses. He doesn't smoke and doesn't consume alcohol. There were no similar episodes in the past. During presentation, his examination findings showed Glasgow Coma Scale (GCS) of 14/15 (E3V5M6). His Blood Pressure on presentation was $210 / 120 \mathrm{mmHg}$. On neurological examination, he had normal motor power and sensation over bilateral upper and lower limbs. Reflexes were intact. However, Cerebellar examination revealed impaired finger nose test. Ataxia was present. But, there was no nystagmus, intentional tremor, slurred speech.

Plain CT Head was performed which showed Right Acute Cerebellar hemorrhage with surrounding edema which was extended up to the fourth ventricle (Figure. 1, Figure. 2, Figure. 3). CT Angiography of Brain was done to rule out any other vascular abnormalities and it inferred normal angiographic findings except hypoplastic Left Posterior Communicating Artery. His CBC, ESR, Coagulation Profile, Immunology, RBS, ECG, RFT and LFT reports were within normal ranges.

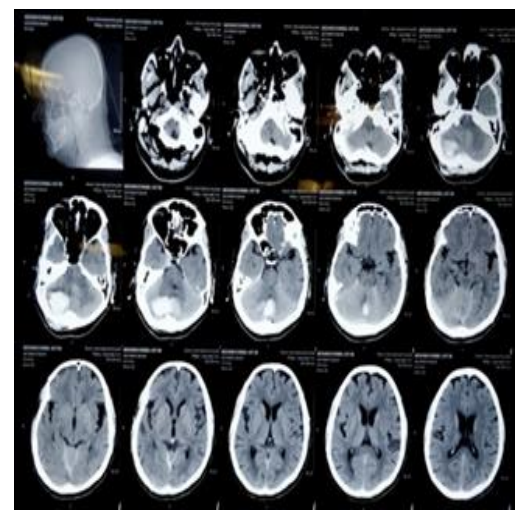

Figure 1: Pre-operative CT Head Plain

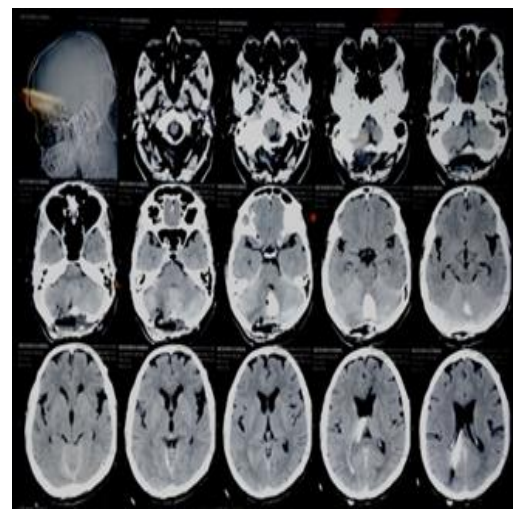

Figure 2: CT Head Plain in 1st Post-operative day

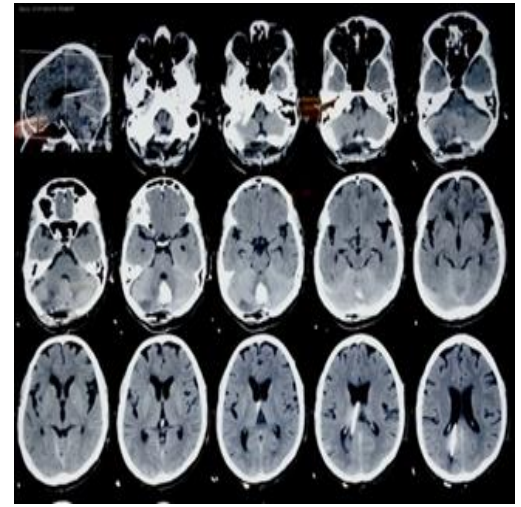

Figure 3: CT Head Pain in 4th Post-operative day before removal of EVD

Then he was immediately planned for surgery and emergency Craniotomy and Evacuation of Posterior Fossa Hematoma with External Ventricular Drainage (EVD) insertion was performed under General Anesthesia. Around 60 $\mathrm{ml}$ of Acute Blood Clots were removed from the Right Cerebellum and EVD was inserted up to third ventricle to prevent possible hydrocephalus due to obstruction at fourth ventricle. He was then 
managed post operatively with IV Antibiotics, IV Fluids, Analgesic, PPI, IV Steroids and Antihypertensive. EVD monitoring was done and it was kept at the level of $15 \mathrm{~cm}$ from tragus of the ear with head elevated at 30 degrees. EVD drain was monitored everyday and drain was clamped on 2nd post op day. CT Head Plain was repeated 24 hours following the clamp, which showed no hydrocephalus, so EVD was removed. The patient showed significant improvement in headache and dizziness following the surgical evacuation and EVD insertion.

\section{Case 2:}

A 67-year Male presented with altered sensorium since 2 days and progressive loss of speech as well as decreased consciousness since 1 day. He had multiple episodes of projectile vomiting. There was no history of complete loss of consciousness, dizziness, paresis or paralysis of any body parts, abnormal body movements, and dizziness. The informant doesn't give a history of recent trauma to the head. The patient is non-hypertensive, nondiabetic and has no other chronic illnesses except he had an episode of raised Blood Pressure, recorded 260/120 one day back for which he was managed in local medical store. He doesn't smoke and doesn't consume alcohol. There were no similar episodes in the past. During presentation, his examination findings showed Glasgow Coma Scale (GCS) of 5/15(E1V1M3). His pupils were Bilateral $2 \mathrm{~mm}$ and Non-reactive to light. Detailed neurological examination couldn't be performed due to poor consciousness level of the patient. He was then immediately intubated in the ER.

Plain CT Head was performed which showed Acute Hemorrhage over the Cerebellar Vermis involving medial part of bilateral cerebellar hemispheres with intraventricular extension resulting in obstructive hydrocephalous(Figure. 4, Figure. 5, Figure. 6). His CBC, ESR, Coagulation Profile, Immunology, RBS, ECG, RFT and LFT reports were within normal ranges.

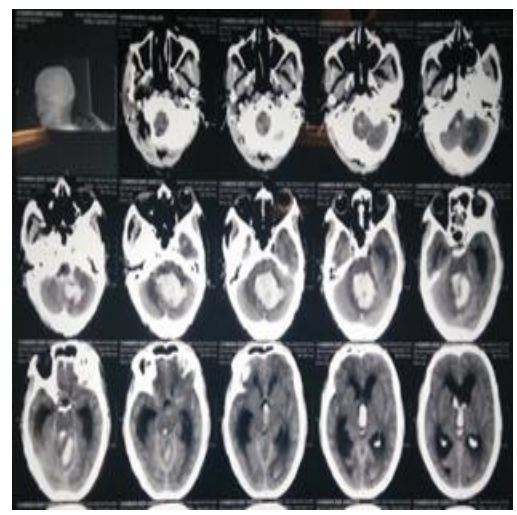

Figure 4: Pre-operative CT Head

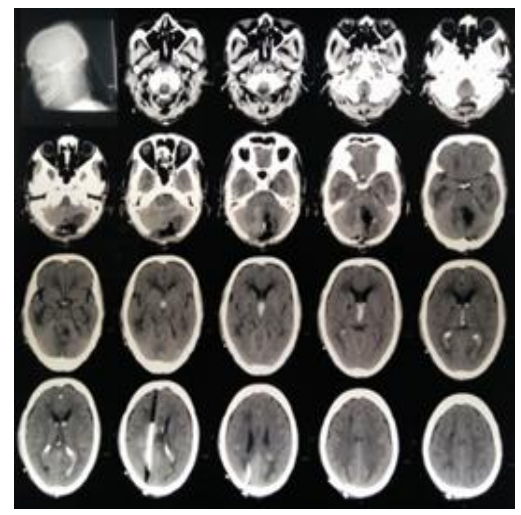

Figure 5: CT Head Plain 3rd Post-operative day

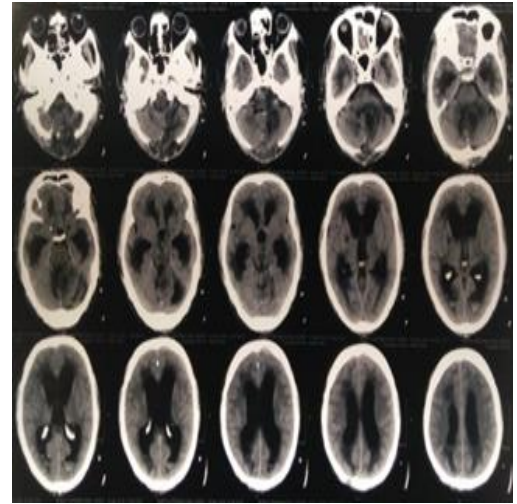

Figure 6: CT Head Plain in 9th Post-operative day

Then he was planned for emergency surgery and underwent External Ventricular Drainage (EVD) insertion with Craniotomy and evacuation of Posterior fossa hematoma under General Anesthesia. Around $40 \mathrm{ml}$ of Acute Blood Clots were removed and 5-10 $\mathrm{ml}$ of blood mixed CSF was drained intraoperative to relieve the pressure. He was then managed post operatively with IV Antibiotics, IV Fluids, Analgesic, PPI, IV Steroids 
and Antihypertensive. EVD monitoring was done and it was kept at the level of $15 \mathrm{~cm}$ from tragus of the ear with head elevated at 30 degrees. Mechanical ventilator support was continued postoperative. Due to poor GCS, he couldn't be extubated so Tracheostomy was done on 3rd post op day. EVD drain was monitored every day and was removed on 9th post op day after repeat CT showed no hydrocephalus. On 13th post op day, CT Head was repeated for poor progression of consciousness level which showed hydrocephalus. So, he was planned for permanent CSF diversion procedure, VP Shunting was done on 14th post op day. Despite all the aggressive management, he had no significant improvement in consciousness level.

\section{Discussion}

The posterior cranial fossa (PCF) is the deepest and most confined space in the skull. It has a limited ability to accommodate an expansion of its contents. ${ }^{1} \quad$ Spontaneous cerebellar hematomas represent 5 to $13 \%$ of all cases of spontaneous intracranial hemorrhage and are associated with high mortality rates of $20-75 \%$ irrespective of the mode of treatment in the pre computed tomography era. Current overall surgical mortality rates remain up to 20 to $50 \%{ }^{2}$

Hypertension is found to be one of the major causes of cerebellar hematoma. A hypertensive cause has been reported to be responsible for 60 to $89 \%$ of cases. Hypertensive cerebellar hemorrhage constitutes 5 to $10 \%$ of all hypertensive intracranial hemorrhage. ${ }^{2}$ Among 52 cases reported by Dinsdale $^{3}$, a previous history of ischemic cerebral infarction was noted in $51.3 \%$ and hemorrhagic infarction in $20.9 \%$ of cases. Both the clinical presentation and subsequent course vary among cases. Unpredictable rapid deterioration in consciousness levels has been recognized. The majority of patients with such decline in consciousness experience the deterioration primarily within $72 \mathrm{hrs}$ after onset. ${ }^{4}$ Acute presentation was observed to be correlated with poor outcomes. ${ }^{2}$

In our report, there was no past history of hypertension in both of our patients. However, Blood Pressure of both patients was significantly elevated on presentation. The first case presented with sudden onset of headache in the right frontal region of head with vertigo. He came to hospital within an hour of onset. However, the second case had an onset of symptoms around 72 hours before the presentation. He had an altered sensorium for 2 days followed by progressive loss of speech and decreased consciousness for a day. He also had multiple episodes of projectile vomiting.

Decision making regarding the appropriate treatment option is crucial for proper management of patients. In most cases, decision regarding surgical treatment is determined by the size of hematoma and other factors, such as the presence of hydrocephalus, the degree of basal cisternal compression, and the location of the hematoma. ${ }^{2}$ The main controversy involves deciding which cases require surgical evacuation of the hematoma versus other options, such as ventricular drainage only or conservative treatment. Furthermore, because the clinical course is variable in some cases, the timing of such treatment should be carefully considered.

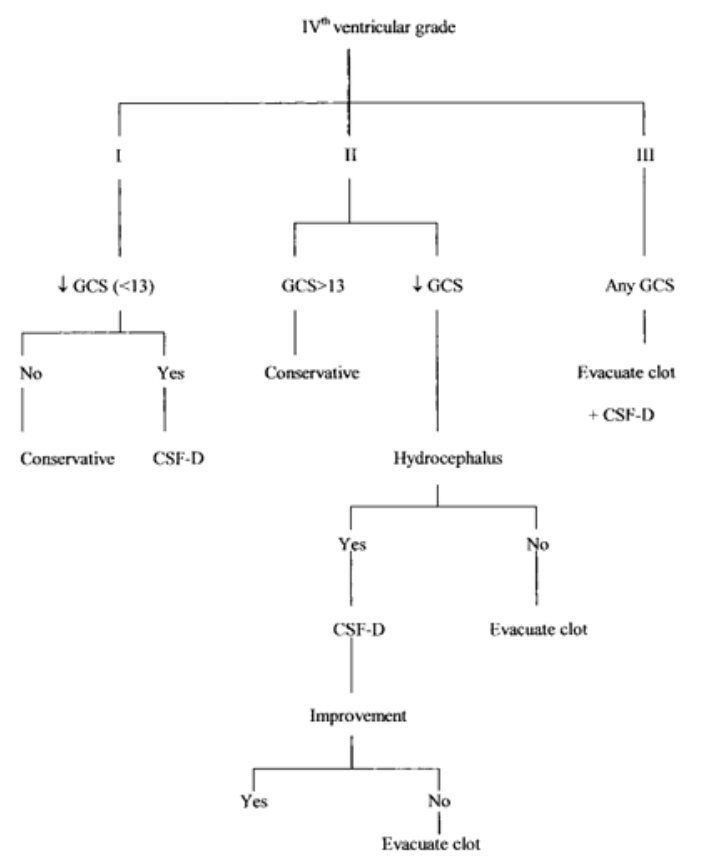

Fig. 7: Protocol scheme for the treatment of spontaneous cerebellar hematomas. CSF-D, CSF/ventricular drainage or shunt ${ }^{2}$

In 2001, Kirollos et $\mathrm{al}^{2}$ formulated a protocol scheme for the treatment of spontaneous cerebellar hematomas (Figure. 7). As a more direct indicator of mass effect within the posterior fossa, the

egneuro, Volume 03, Issue 01, 2021 
configuration of the fourth ventricle was noted and a grading system was based on the CT appearance of the fourth ventricle, dividing it into three grades. Grade I, normal size and configuration, located in the midline (if intraventricular hemorrhage is present, cerebrospinal fluid [CSF] is still visible in the fourth ventricle); Grade II, partially compressed or distorted, shifted to the contralateral side (in cases of unilateral hematomas); Grade III, complete obliteration, with anterior shift distorting the brainstem and obliterating the pre-pontine space (even if the fourth ventricle is partially compressed). ${ }^{2}$

According to the protocol, our first case was included in Grade II and the second case in Grade III. Both patients underwent CSF diversion (EVD insertion) and evacuation of Posterior Fossa Hematoma under General Anesthesia. Case 1 had a significant improvement in consciousness level, symptoms and post op CT scan showed decreasing hydrocephalous and mass effect due to clot evacuation. However, there was no satisfactory improvement in consciousness level of Case 2, despite having comparatively better findings in the post op CT scan. He was under mechanical ventilation and had undergone Tracheostomy on 3rd post op day due to no improvement in GCS and continued mechanical ventilator support. He had to undergo permanent CSF diversion procedure (VP Shunting) due to recurrence of hydrocephalus.

There has always been a personal preference regarding the conservative or surgical management especially when it comes to the borderline cases. Even when surgery is indicated, controversy exists regarding whether ventricular drainage only, evacuation of the hematoma, or both procedures should be performed. Some recommend the ventricular drainage as the only initial procedure for all cases, whereas others opt for evacuation of hematoma whenever surgery is indicated.

But, choice of procedure is not the only factor that helps in early improvement in the condition of patient. The time of presentation and intervention plays a pivotal role in overall improvement regardless of procedure done. Both of our cases belonged to similar age group, had similar hematoma in the posterior fossa and had undergone the similar surgical procedure. However, Case 1 showed significant improvement in his condition the following day of surgery whereas; Case 2 developed multiple post op complications and had to undergo further surgeries following the first one. Case 1 presented to the hospital and underwent surgical intervention within 6 hours of onset of symptoms whereas Case 2 was intervened $72 \mathrm{hrs}$ after the occurrence of his symptoms.

\section{Conclusion:}

Posterior cranial fossa holds the crucial parts of brain which is responsible for carrying out the vital bodily functions. When a part of it gets compromised, the brain goes through various adaptation processes in the beginning to counteract the stress it is put through. So, it is of utmost importance to act soon to prevent diffuse and possible irreversible damage.

Although surgical intervention itself might have their own risks and complications, the patient condition, radiological findings and the course of progression gives an idea regarding the type of procedure required.

The presentation and course may vary in different cases as evident by the two cases discussed above; however the treatment modalities and time of intervention might create a huge difference in the recovery and prognosis of one of the life threatening intracranial hemorrhages.

\section{References:}

1. Luney MS, English SW, Longworth A, et al. Acute Posterior Cranial Fossa Hemorrhage-Is Surgical Decompression Better than Expectant Medical Management? Neurocrit Care. 2016; 25(3):365-370. doi:10.1007/s12028-015-0217-7

2. Kirollos RW, Tyagi AK, Ross SA, et al. Management of spontaneous cerebellar hematomas: A prospective treatment protocol. Neurosurgery. 2001; 49(6):1378-1387. doi:10.1097/00006123-200112000-00015

3. DINSDALE HB. Spontaneous Hemorrhage in the Posterior Fossa. Arch Neurol. 1964; 10(2):200. doi:10.1001/archneur.1964.00460140086011

4. Fisher CM, Picard EH, Polak A, Dalal P, Ojemann RG. Acute hypertensive cerebellar hemorrhage: Diagnosis and surgical treatment. J NervMent Dis. 1965; 140(1):38-57. doi:10.1097/00005053-196501000-00004

egneuro, Volume 03, Issue 01, 2021 